\title{
MOORECUBE: A SCALABLE AND FLEXIBLE ARCHITECTURE FOR CLOUD COMPUTING DATA CENTERS ON MULTI-PORT SERVERS
}

\author{
AREZOO JAHANI AND LEYLI MOHAMMAD KHANLI *
}

\begin{abstract}
Networks provide the infrastructure of cloud computing data centers. Servers in data centers grow to respond to the growing user demands. Data center scalability and flexibility in the use of multi-port servers is a challenging issue. Heretofore, no method has been considered for scalability and flexibility issues. This paper proposes a new architecture called MooreCube that can increase network scalability and decrease network diameter as well as increase flexibility. MooreCube is a scalable and flexible architecture that each multi-port server directly connected to other servers via bi-directional links, without using any switch. Furthermore, MooreCube is a recursively defined architecture that uses Moore graph as Building Block (BB) structure and uses the hierarchical structure to meet high scalability. The paper proposes a multipath routing to increase fault tolerance and decrease links burden. MooreCube architecture compared with other switchless architectures that use reserved ports to increase scalability. The simulation results show MooreCube increase scalability and flexibility along with decrease the diameter of the network.
\end{abstract}

Key words: Data center network, Cloud computing, Network architecture, Multi-port servers, Scalability, Multipath routing.

AMS subject classifications. $68 \mathrm{M} 14,68 \mathrm{M} 10$

1. Introduction. Cloud computing uses data center networking (DCN) as an infrastructure to provide services [1]. The powers of data centers should be enhanced with an increase in the service request and computational, storage and processing requirements $[2,3]$. Therefore the architecture of data centers must be scalable and loosely as possible to add and/or subtract server/s to data centers $[4,5]$. In addition, to exposure better service, the architecture of data centers should provide communication between servers that is possible by high-speed links, bilateral and optimized routing [6].

Adding new server/s to data center has some Issues. The first issue is the network flexibility that possible adding any new server to the data center. The former architectures have limitations in adding new servers. Because most of them use the reserved port and when the reserved ports completed, adding any new server is not possible [3, 7]. Cost is the second issue. Adding new server/s has cost like the price of adding the new link, switch, and router. Although it has some other issues like inefficient network development which limit the use of all server capacity because of inefficient development $[8,9]$. Therefore considering these issues will help in designing new data center architecture. Design goals of the data center are; scalability, fault tolerance, latency, network capacity, simplicity, flexibility, and network configuration that will describe in detail $[2,32]$.

- Scalability: Ease of adding new server/s to data center networks. Scalability shows that adding any new server should not modify the DCN architecture (topology).

- Fault tolerance: The DCN should be accessible in presence of fault. Mostly, the lack of alternative routes and servers lead to irreparable damage.

- Latency: Delay in sending and receiving the message has a direct relation with network diameter (number of steps between source and destination). So latency in DCN architecture is important. The network diameter is most important factor in architecture and should not increase with enhancing network scalability.

- Network capacity: Total network capacities such as server's CPU, hard and link bandwidth should be accessible and using all network capacity should be possible.

- Simplicity: Ease of creating architecture is an important issue that must be considered. Use of former routing would be possible if the DCN architecture not modified in adding any new server/s.

- Flexibility: Flexibility can be defined in two ways. Network Flexibility and flexibility in using multiport servers. Network Flexibility means the possibility of using small and very large architecture scale in presence of all network features. Flexibility in using multi-port servers as the name implies is possible using servers with a different number of ports.

\footnotetext{
${ }^{*}$ Faculty of Electrical and Computer Engineering, University of Tabriz, Tabriz, Iran. (a.jahani@tabrizu.ac.ir, l-khanli@ tabrizu.ac.ir)
} 
- Modularity: Recursive architecture has modularity concept. This kind of architectures, use a basic structure that repeated with adding any new server and known as modularity architectures.

Data center architectures include in three general categories: switch-centeric architecture, server-centeric architecture and switchless architecture $[2,6,7]$. First two categories have the switch in their architecture to connect servers. The only difference between them is the location of network routing. In a switch-centeric architecture, the switch is responsible for message routing in the network. But in server-centric architecture, servers set up network routing. The last one not uses the switch in DC architecture and connects servers directly using multi-port servers [2]. Using any switch in DC architecture has some advantages like; reducing the cost and increasing the network efficiency.

This paper proposes MooreCube as a new architecture that not use any switch to connect servers. This new architecture uses multi-port servers to connect servers directly with bi-directional links. MooreCube use Moore graph as a basic Building Block (BB) and hierarchical structure to increase network scalability. Moore graph is a regular graph that is known with degree and diameter $[12,13]$. The paper uses multipath routing to increase fault tolerance and reduce the burden of links [14, 15].

The reminder of paper is organized as follow; section 2, studies previous solutions. Section 3 describes MooreCube architecture. Section 4, shows multipath routing and in continue, section 5 evaluates the MooreCube strategy and at last, section 6 express conclusion and future works.

2. Related works. Researchers are trying to find a way to properly connect servers to each other to reduce the network diameter and increase network scalability. Many researchers have been done activities in this area. In general, the DCN architectures divided into three categories include; switch-centric architecture, server-centric architecture, and switchless architecture. In switch-centric architectures, the switch is responsible for routing and this architecture mostly uses optical interconnections to send and receive messages [7, 11]. The first switch-centric architecture was proposed in 2008 called FatTree [16]. FatTree uses some switches and servers as Building block and increases the number of switches with adding any new server/s. FatTree has the three-level architecture that the servers are at the lowest level and switches are in two high levels of architecture. Depending on the number of servers that are at the lowest level, switches have the port. These layers are connected to each other in form of tree. However, each routing between servers in lowest level needs to use existing switches in the high level. In the worst case, all the pairs of servers are linked together. So, using the switches in this way, make switches as a bottleneck. Portland architecture [17], is another architecture that uses FatTree with a new send/receive protocol [18]. VL2 is another architecture that focuses on the use of server capacity and facilitates the routing with using of flat addresses. SWDC [23], Poincare [22], Scafida [21], S2 [20], Jellyfish [19] and RingCube [7] are also switch-centric architectures. All of the solutions use switch beside server in DCN architecture.

Server centric architecture also has a switch but use the servers as a router. DCell [24], BCube [25], Ficonn [26], FlatNet [27], HCN \& BCN [28], Dpillar [29], SWCube [30], FleCube [31, 32], DCube [33], Fsquare [34] and sprintNet [35] are server-centric architecture. Using the switch in this kind of architecture may lead to bottleneck and increase cost and scalability of the network. The third category of DCN is switchless architecture whose does not use any switch to connect servers. But servers are directly connected to one another by the bidirectional link. Do not use the switch in DC architecture reduce the cost and increase the network efficiency [35]. Because in the absence of switch, the cost, and power that are needed to switch cares, are stored. Also if we do not have the switch, switches will not have downtime and network fault tolerance will be greater. Delay in the switch is also another reason for not using the switch. Because each switch has few microsecond delay to send and receive data. We can use the server instead of switch in DCN and increase network scalability [33].

CamCube [36], Smallworld [23], NovaCube [37] and FleCube [2] are switchless architecture. CamCube uses three-dimensional Torus structure for connectivity between the servers. This architecture needs 6-port servers in all circumstances. The Torus-3D indicated with k-array-3-Cube that the number of servers (S) computed with Eq. (2.1) and Diameter (D) computed with Eq. (2.2).

$$
\begin{gathered}
S=k^{n}=k^{3} \\
D=\sqrt[3]{(}\left(k^{n}\right)=\sqrt[3]{(}\left(k^{3}\right)=k
\end{gathered}
$$


In Eq. (2.1), $S$ is the number of servers and $\mathrm{k}$ indicates the number of servers required in each row of CamCube architecture and $n$ is the dimension of Torus architecture (in CamCube $n=3$ ). In Eq. (2.2), $D$ is the network diameter. Smallworld is another architecture that presented to reduce the diameter of CamCube. This architecture in order to reduce network diameter, add a random number links to CamCube architecture. Results show that the network diameter is reduced by adding additional links [23], but the number of ports on each server must be greater than 6 and we cannot definitely determine needed ports on each server. Also, the randomly added links are only shortest path and may become a bottleneck. NoveCube is another architecture that adds new links but not randomly. This architecture found pair servers that are far from each others and then with adding new link connects them. So the diameter of the network will be decreased. But this approach also has the problems of previous work and needs more ports. SprintNet has the new way to connect servers to each other without use of switches [33]. This method is flexible and can be implemented with multi-port servers independent of the number of ports. The problem with this solution is its limited scalability due to server port number. For example, with 3-ports servers cannot connect more than 42 servers directly. Our presented MooreCube architecture solves port and diameter issue and is scalable and flexible with any number of server ports.

3. MooreCube architecture. In this section, physical structure and characteristics of MooreCube are expressed. In order to measure the diameter of the network, one-way routing described below to find the shortest path between servers.

Physical structure. MooreCube architecture uses multi-port servers in its structure. Multi-port servers connected to one another by bidirectional links. This architecture does not use any switch to connect servers.

Moore graph. MooreCube uses recursive structure and multi-port servers. The basic Building Block (BB) in this architecture is Moore graph. Moore graph is one of the most famous mathematical graphs, and these graphs may be used in any number of vertices $[12,13]$. In fact, Moore graph is a k-regular $(k>2)$ graph that is displayed with two variable $n=(v, g)$. $v$ represents the degree vertices in the graph and $g$ is waist size of graph or a complete cycle (without duplicate vertices) of the graph. With these two variables, we can calculate the number of vertices in the graph (n) using Eq. (3.1).

$$
n(v, g)= \begin{cases}\left(1+(v-1)^{((g / 2)-1)}+v \sum_{r=0}^{(g-4) / 2}(v-1)^{r},\right. & \text { if } g \text { is even } \\ 1+V \sum_{r=0}^{(g-3) / 2}(v-1)^{r}, & \text { otherwise }\end{cases}
$$

In Eq. (3.1), $n$ is the number of vertices in the graph. $v$ is vertices degree and $g$ is waist of the graph. Fig. 3.1 outlines a number of Moore graphs with different vertices degree.

As mentioned in Fig. 3.1, the graph $(n=4)$ is a Moore graph with the degree $(v=3)$ and waist $(g=3)$ that can be computed with Eq. (3.1). Fig. 3.1 indicates various Moore graphs with different vertices [38]. With compliance Moore graph on DCN, graph vertices will express the servers and edges will express link between servers. In this case, $v$ (the degree of Graph) will indicate the number of needed ports (in BB) and $g$ will indicate network girth. Calculate the network diameter with the use of $g$ will be described in the following.

Moore graph can be different values for $v$ and $g$. also the number of Moore graphs generated for each pair $(v, g)$ can be more than one. For example, for the pair $(3,5)$ we can generate nine different Moore graph. Moore graph shows the flexibility for any environment and can be used with any wire length.

Some of the specific values of $v$ and $g$ graphs created and has the certain name that was given to them by the mathematical researchers. Table 3.1 shows some of these names and specific Moore graphs.

Peterson graph is a special case of Moore graph with the vertex as $v=3$ and waist as $g=5$. The pair $(3,5)$ can generate nine different Moore graphs as shown in Fig. 3.2 and has 10 servers in total that can be computed in Eq. (3.1) $(n(v, g)=10)$.

According to what is shown in Fig. 3.2, Peterson graph in all of 9 cases has the diameter equal to 2. This means that any two vertices that are far from each other have distance equal to 2 . So we need a way to calculate the diameter of network using girth.

Theorem. Diameter of each regular graph is $D_{\text {Basic }}=\lfloor g / 2\rfloor$, if $g$ indicates waist length of the graph. ( $D_{\text {Basic }}$ indicates the diameter of graph.)

Proof: Waist length in each graph is equal to the number of edges from each vertex to itself through the unique vertices. Since the graph is regular, this length for all graph vertices will be equal. For each vertex, 


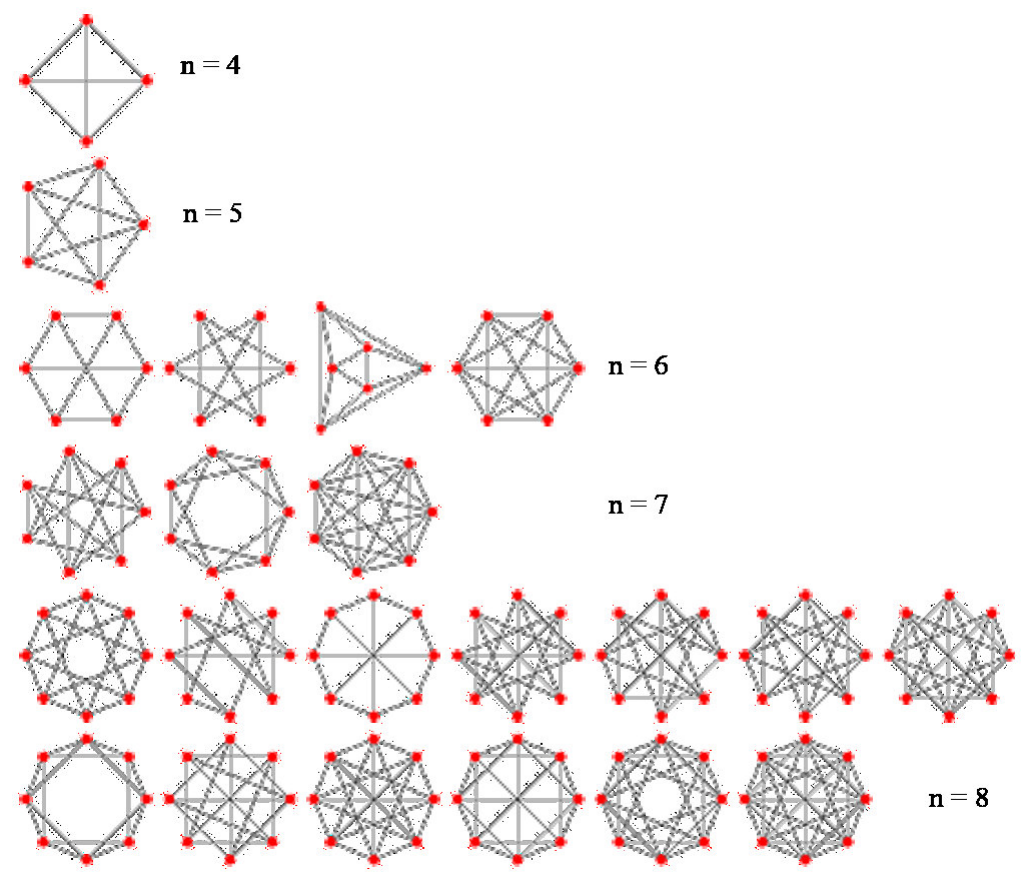

FIG. 3.1. Moore graphs with different number of vertices

TABLE 3.1

Specific Moore graph

\begin{tabular}{|l|l|}
\hline $\mathbf{( v , g )}$ & Name of Moore Graph \\
\hline$(3,5)$ & graph Petersen \\
\hline$(3,6)$ & graph Heawood \\
\hline$(3,8)$ & graph Levi \\
\hline$(4,6)$ & graph Wong \\
\hline$(5,6)$ & graph order-4 generalized triangle \\
\hline$(7,5)$ & graph Hoffman-Singleton \\
\hline
\end{tabular}

a path that shows the number of edges from the vertex to itself has passed the farthest vertex of the graph and returns to the source vertex. So the waist graphs show a complete cycle through the farthest vertex of the graph. Obviously, the length of the waist can be halved to calculate the farthest vertex. As mentioned in Fig. 3.2(a), the distance from each vertex to farthest vertex in the graph is 2 . So for different values of $g$, the network diameter is equal to low $g / 2$ and prove that $D_{\text {Basic }}=\lfloor g / 2\rfloor$.

Opposite Theorem. For every regular graph with $D_{\text {Basic }}$ diameter, waist length $g$ calculated as $2 D_{B a s i c}$ or $2 D_{\text {Basic }}+1$.

Peterson graph has vertex degree equal 3. This means if basic BB structure degree was 3 , we need to three ports of multi-port servers. For network scalability, we can connect BB structures with links and in a hierarchical structure. Suppose the first case of Peterson graph is used as the basic structure of architecture. This basic structure has 10 servers that can be put $k$ number of these structures over each other as showed in Fig. 3.3. For example, if we want to have 30 servers, we should 3 BB over each other.

As mentioned in Fig. 3.3, to scale the network, two extra port of each server is required to connect BB structures on top and bottom to each others. The proof shows that the number of ports per server in MooreCube is $v+2$. $v$ ports to connect to other servers in same $\mathrm{BB}$ and 2 port to connect to other $\mathrm{BB}$ on top or bottom of that. 


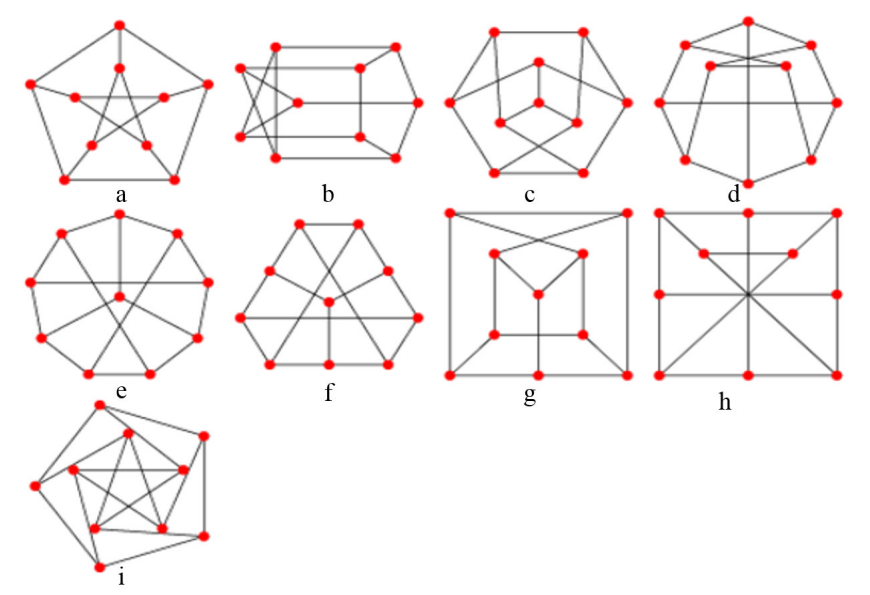

FIG. 3.2. Moore graphs generated for the pair (3,5)

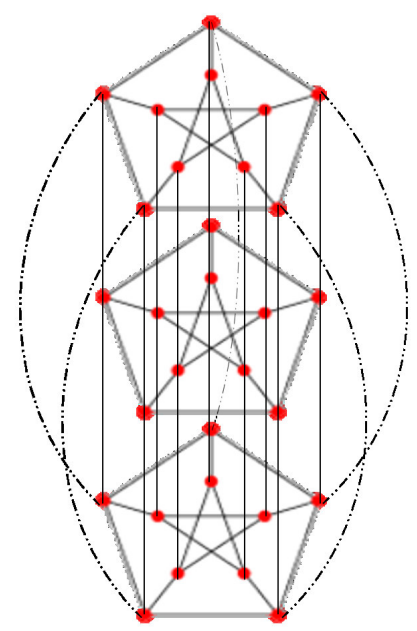

FIG. 3.3. Peterson graph used as basic for a structure with 30 servers

However, when mapping the network with Moore graph, at first we need to know how many ports are required for each server. It should be noted that the proposed architecture is capable to build with any number of ports which is an advantage and Indicator of Scalability. With having the number of ports that are required to each server, the basic structure can be found ( $v=$ number of ports- 2 ). By choosing variable $v$, we can calculate needed servers for any value of $g$ and selected the best and the most accepted structure. With having $g$ and $v$, the BB structure will be determined by Eq. (3.1). Then with dividing the whole needed servers (S) to the number of servers in each $\mathrm{BB}$ can compute the number of BB that is required that is indicated in Eq. (3.2).

$$
k=S / n(v, g)
$$

In Eq. (3.2), $k$ is the number of BB and $S$ is the whole number of servers and $n(v, g)$ indicates the number of servers in BB. As shown in Fig. 3.3, the whole diameter of the network is different from BB diameter $\left(D_{\text {Basic }}\right)$. Therefore it is necessary to calculate the overall diameter of the network. Overall diameter equal to the diameter of the BB network, plus the number of steps required to go from one structure to the farthest structure. Because every time there is a link that connects the two upper and lower structure, so pairs of far 
TABLE 3.2

Calculate best waist length with degree $=3$

\begin{tabular}{|l|l|l|l|l|l|l|}
\hline$v$ & $g$ & $n(v, g)$ & $S / n(v, g)=k$ & $S$ & $\begin{array}{l}D_{\text {Basic }} \\
\lfloor g / 2\rfloor\end{array}$ & $\begin{array}{l}D_{\text {Total }}= \\
D_{\text {Basic }}+ \\
\lfloor/ 2\rfloor\end{array}$ \\
\hline 3 & 1 & 1 & 30 & 30 & $1 / 2$ & 16 \\
\hline 3 & 2 & 2 & 15 & 30 & 1 & 8 \\
\hline 3 & 3 & 4 & 7.5 & 30 & 1 & 4 \\
\hline 3 & 4 & 6 & 5 & 30 & 2 & 4 \\
\hline 3 & 5 & 10 & 3 & 30 & 2 & 3 \\
\hline 3 & 6 & 14 & 2.14 & 30 & 3 & 4 \\
\hline
\end{tabular}

structures has a length of $\lfloor k / 2\rfloor$. The overall diameter of the network is calculated by Eq. (3.3).

$$
D_{\text {Total }}=D_{\text {Basic }}+\lfloor k / 2\rfloor
$$

In Eq. (3.3), $D_{\text {total }}$ is the overall diameter of the network, though $D_{\text {Basic }}$ is BB diameter and $\mathrm{k}$ is the number of BB.

Practical example. Suppose we have 30 servers and each server has 5 ports. In order to find the number of required BB, first, we should compute the degree of the basic graph. Because the servers have five ports, two ports of them will be reserved to connect BBs together. Then we have three ports for BB graph and $v=3$. For $v=3$, we can consider different waist lengths. Table 3.2 shows the number of servers used in any structure with different $g$. in this table, $D_{\text {Total }}$ can be calculated for each $g$. so the most appropriate structure will be selected. It is obvious the row with lowest $D_{\text {Total }}$ in the table is the best structure. But no need to calculate all values for all of $g$. because $g$ and $D_{\text {Total }}$ has a regular trend. $D_{\text {Total }}$ will be reduced with increasing $g$ and then enhanced when passed the best value of $g$. this regular trend helps us to choose the most suitable amount of $g$. in Table 3.2, the lowest of $D_{\text {Total }}$ belong to the fifth row. So the value of $g=5$ will be chosen that has 10 servers in the structure of the BB.

According to Table $3.2, D_{\text {Total }}$ with different $g$, will be declined at first and then with passing the best value of $g$, start to enhanced. So choose the most optimal value for the variable $g$ easily computed with the comparison of values in the $D_{\text {Total }}$ column.

4. Routing. MooreCube structure constructed of many BB and each BB constructed of many servers. Routing in each $\mathrm{BB}$ can be done by broadcast shortest path (BSP) algorithm. It is essential to note that routing algorithms run once before DCN running. So DCN uses the results of routing algorithm. In fact, all routes should be saved in routing tables for future use. So the complexity of routing algorithm will not have any effect on the routing between data centers and most of the routing algorithm can be easily used for routing. Finding the shortest path with BSP is very simple algorithm where each server in order to find the shortest route to the destination server, first sends a message includes destination server name to all neighbors. The neighbors who received this message sends the message to their neighbors. This process continues and if the message received by destination server, process terminated and messages come back to the source server and collect the intermediate servers as a route from source to destination. The source has received several messages from different directions to pass each of which have the shortest path as the best path is selected. Routing in each pair of $\mathrm{BB}$ is carried out by a link between them. Each server who wants send a message to a server in another BB, there are two choices:

- Use the link that connects the source to destinations neighbor (in destination BB).

- Use the link that connects destination to sources neighbor (in source BB).

Routing procedure between each source and the destination indicated in the algorithm 1.

As indicated in the algorithm 1, this algorithm first check if source and destination are the neighbor. If they were the neighbor, the link between them returned as route (rows 1-3). Otherwise, select one of the links between two $\mathrm{BB}$ as the intermediate link in order to find the shortest path (row 4). Then depending on which link is chosen, calculate the shortest path using BSP algorithm (rows 5-9). 


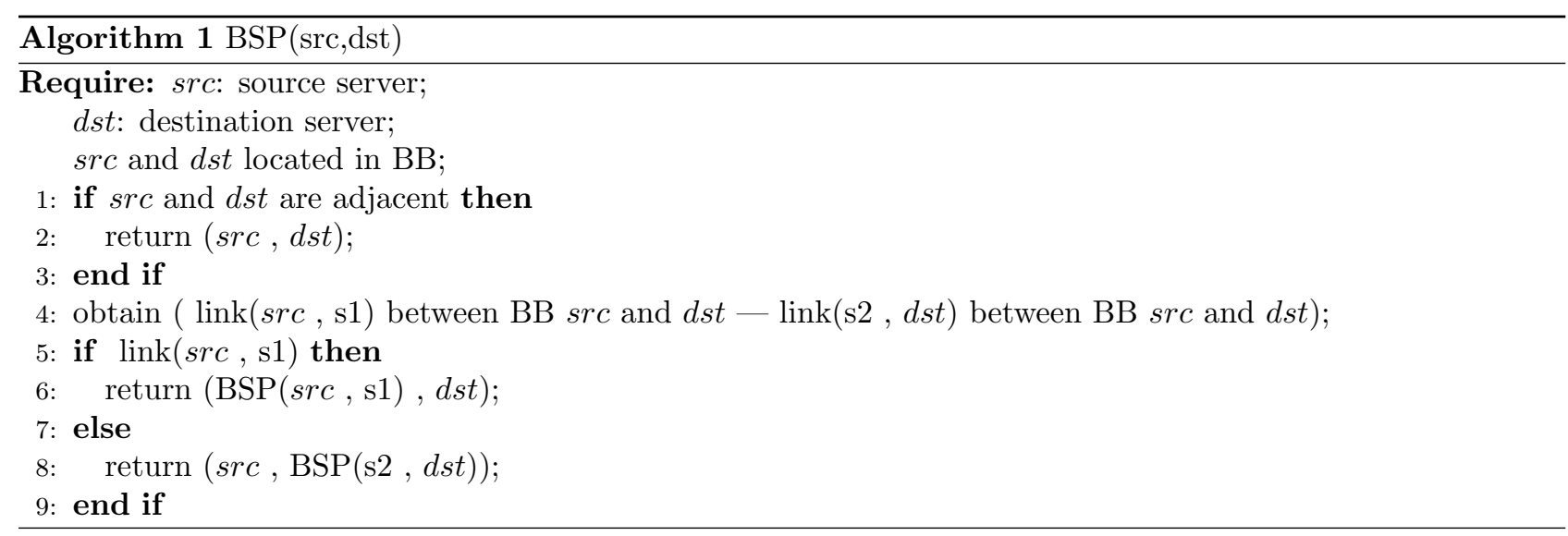

\section{MooreCube features.}

- Scalability: MooreCube scalability enhances by increasing the number of ports (server ports) as well as network diameter. According to Eq. (3.2), the number of required BB is equal to $k=S / n(v, g)$. So the number of servers depends on $k$ and the number of ports in BBs servers. As a result, the total number of servers in each layer of the hierarchical structure will be calculated by Eq. (4.1).

$$
S_{k}=k \times n(v, g)
$$

Eq. (4.1) indicates the number of possible servers in each layer of hierarchical structure k. S_k is the number of servers for all $\mathrm{k}$ layer. $\mathrm{K}$ is the number of required $\mathrm{BB}$ and $n(v, g)$ indicates number of servers in each BB. Increase the number of servers with increasing variable $k$ in Section 5 and will be shown in experiments related to scalability.

- Flexibility: Flexibility is one of the most important features in MooreCube architecture. The purpose of the flexibility is using architectural with the different number of ports. MooreCube is capable of using multi-port servers in architecture. Although the architecture FleCube [2] also was dealt with this issue in 2015. But FleCube focused on only flexibility and scalability was not adhered to. For example, when using FleCube architecture and 3-port servers we cannot have more than 42 server connection and adding more servers is not possible in this architecture. Therefore, the proposed solution unlike previous methods focusing on both flexibility and scalability.

- Network diameter: Network diameter indicates the number of steps from a source server to farthest server on the network. According to section (3.1.1) and proved theorem we know that the network diameter can be determined by variable $\mathrm{g}$ and the overall diameter of the network is $D_{\text {Total }}=D_{\text {Basic }}+$ $\lfloor m / 2\rfloor$.

- Bidirectional bandwidth: Network bandwidth indicates data transfer rate on network connections. The criterion is the most important criteria for determining the speed of a network. Accordingly, bi-directional bandwidth indicates data transmission rate by network connections. MooreCube architecture has the bidirectional bandwidth. Eq. (4.2) shows the bidirectional bandwidth of MooreCube architecture.

$$
v+2 \leq \text { bidirectional }- \text { bandwidth } \leq n(v, g)
$$

In Eq. (4.2), $v$ is the number of ports in each BB.

- Theorem: Bidirectional bandwidth in MooreCube structure is accordance with Eq. (4.2).

Proof: To calculate the bidirectional bandwidth, the network should be divided into two equal part. The simplest way to divide MooreCube is to divide it into two separate BB that have equal servers. In this case, the link between these two parts will be equal to the number of servers in each BB unit. all servers of two connected BB have a link together. So at best case, bidirectional bandwidth is equal to 
the number of servers in BB. In the worst case, there is only a BB should be divided into two equal parts. In dividing time, if the number of servers is even, we can divide them simply in two parts and it is clear that only $v+2$ link between these two parts can connect parts to each others. Otherwise, if the number of servers is odd, we can omit one of them and use the earlier case to calculate network diameter.

- Links and cost: Given that more links in any architecture, increase fault tolerance on the network. But connecting any two servers is not possible. Because it led to wiring problems and eliminate the flexibility and scalability of the network. So we should make a trade-off between the number of links and scalability, flexibility and wiring problems. The number of links should be low as much as possible and next to it the flexibility and scalability of the network should be high as much as possible. MooreCube has the simple structure and adding any new server do not need much cost, because not need any switch and do not change BB structure. In addition, the number of links in each BB is less than the number of links in a complete graph and this is easily measurable by the number of used server ports.

- Number of parallel disjoint paths: According to section 4 of this article, the following is stated, it can be concluded that the number of parallel disjoint paths is equal to $v+2$. The proof is given below.

- Theorem: The number of the parallel disjoint path in MooreCube is $P k=v+2$ (if $P k$ be the number of the path).

Proof: Each server like $a$ and $b$ has set of neighbors such as seta and setb. We know $\mid$ set $_{a}|=|$ set $_{b} \mid=$ $v+2$. These two elements can have three types of communication: (1) $a$ and $b$ are neighbor and $a \in$ set $_{b} a n d b \in$ set $_{a}$. So $a$ and $b$ has a link that connects them. (2) $a$ and $b$ have a common neighbor. So the number of common neighbors is $\mid$ set $_{a} \bigcap$ set $_{b} \mid$. In this case, $a$ and $b$ has a path with the transition from middle neighbors. (3) Each server in seta belong to a BB and setb belong to BB too. These two $\mathrm{BB}$ can be equal or not, in every case $a$ and $b$ have a link/path and can connect to each other. So prove that a path is between $a$ and $b$ with the transition from middle neighbors and $P_{k}=v+2$.

5. Multipath routing. In order to have several paths which are parallel and disjoin, disjoint parallel path (DPP) is provided. DPP can select multiple paths between each pair of source and destination which has less congested links and uses all link capacity. So using DPP, increase bi-directional bandwidth efficiency and protect network failure.

DPP algorithm uses BSP to find disjoint parallel paths. But using BSP, may led to loop or common link paths in the network and we know this kind of paths which have common link or loop can cause network congestion and failure. So all of the parallel paths should be disjoint with any common links. In order to have such routes, we should change slightly BSP algorithm. This modified algorithm is shown CBSP. CBSP has three entrance; source, destination and limitation set that includes some server and CBSP should find the shortest path between source and destination without violating from limitation servers. The procedure is like that section (2.1.3) but source server should send limitation set when sending a broadcast message to their neighbors. Each intermediate server with receiving a message, check the content of the collection, if its id is found within the limitation set, it terminated and not send the broadcasting message. Algorithm (2) indicates DPP with use of CBSP (improved BSP).

Algorithm 2 calculate the disjoint parallel path for each pair of source and destination. This algorithm creates set $u$ with neighbor servers id of the source server. Then if the source and destination server are the neighbor, return their link (rows 1-3). Otherwise, add source $i d$ to limitation set and send broadcasting the message (row 4). Each server can have parallel path depend on a number of ports, therefore for every server calculate paths based on their neighbors (row 5-8).

Then all intermediate servers added to limitation set (row 9) in order to next parallel path do not use this server as middle servers.

6. Evaluation results. This section evaluates MooreCube with three kinds of tests includes network diameter, flexibility, and scalability. In all tests, the proposed MooreCube and other compared solutions implemented in MATLAB software, version 7.14.0 (R2-15a) on a computer with Intel Core i5 Duo 2.53 GHz, 4 GB Memory and Windows 7 x86 enterprise.

MooreCube compared with CamCube [34] and FleCube [2] solutions. CamCube uses three-dimensional Torus structure and focuses on scalability and FleCube focus on flexibility. 


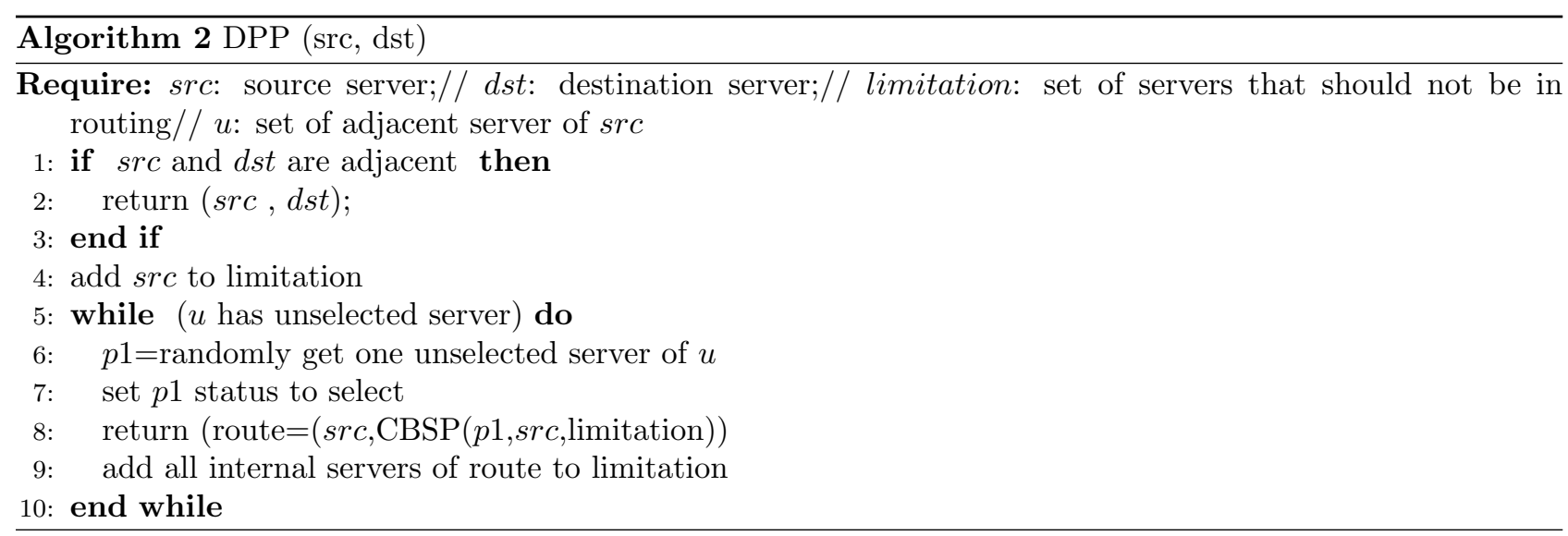

In following this section, an experiment done for measure delay in sending and receiving message using DPP and results are given below.

6.1. Network diameter. In this experiment, the diameter is calculated with increasing number of servers. Server numbers are from 10 to 105. In each stage, based on a number of servers, appropriate network diameter is selected. The results are shown in Fig. 6.1.

As shown in Fig. 6.1, when 6-port servers increase, network diameter in CamCube increase exponentially. FleCube has better network diameter but focuses only on flexibility. Our proposed MooreCube has lowest network diameter. Because MooreCube uses Moore graph and can use any server ports and can have scalability using the hierarchical structure.

6.2. Flexibility. Flexibility means useing of architecture with any number of ports in the server and the architecture must not depend on port and should be usable regardless of server ports. MooreCube is constructive with any number of server ports. This experiment shows MooreCube flexibility in compress of FleCube and CamCube. This experiment measures the maximum number of servers that each strategy can connect with a specified port number. The experiment results are shown in Fig. 6.2.

As indicated in the Fig. 6.2, CamCube use only 6-port servers and can connect any number of servers with

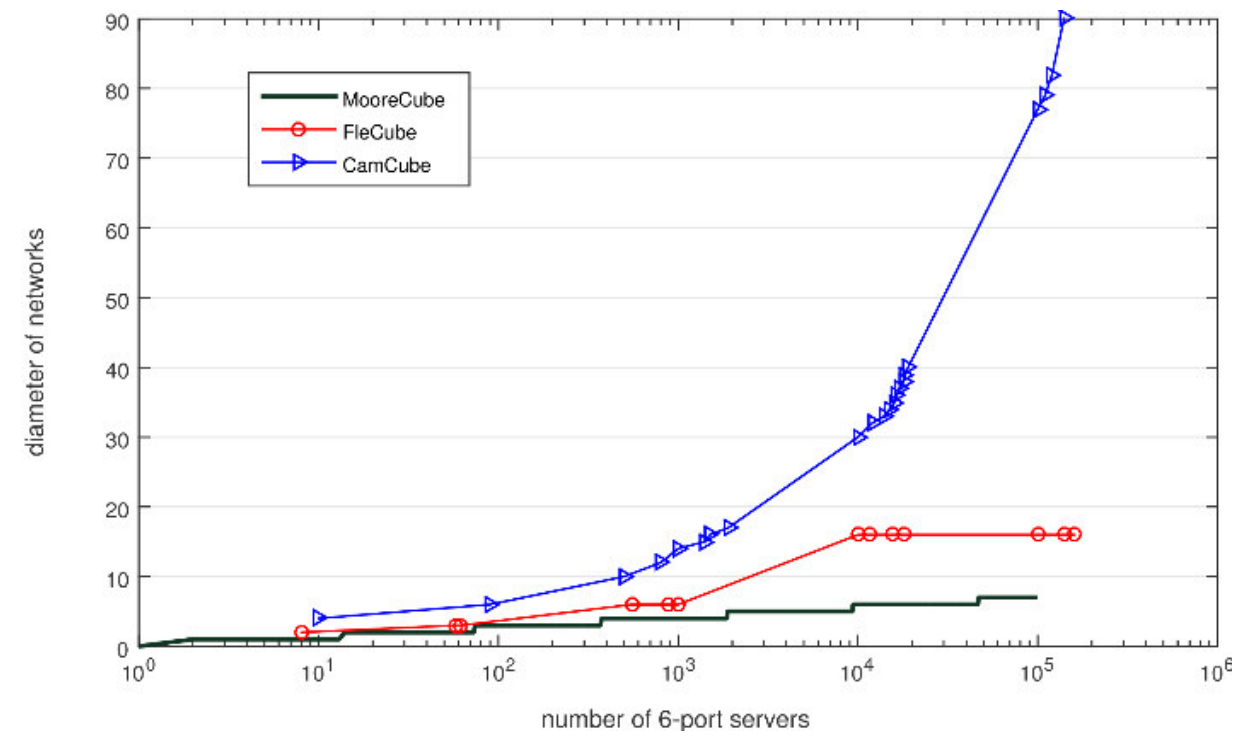

FIG. 6.1. Network diameter (6-ports server) 


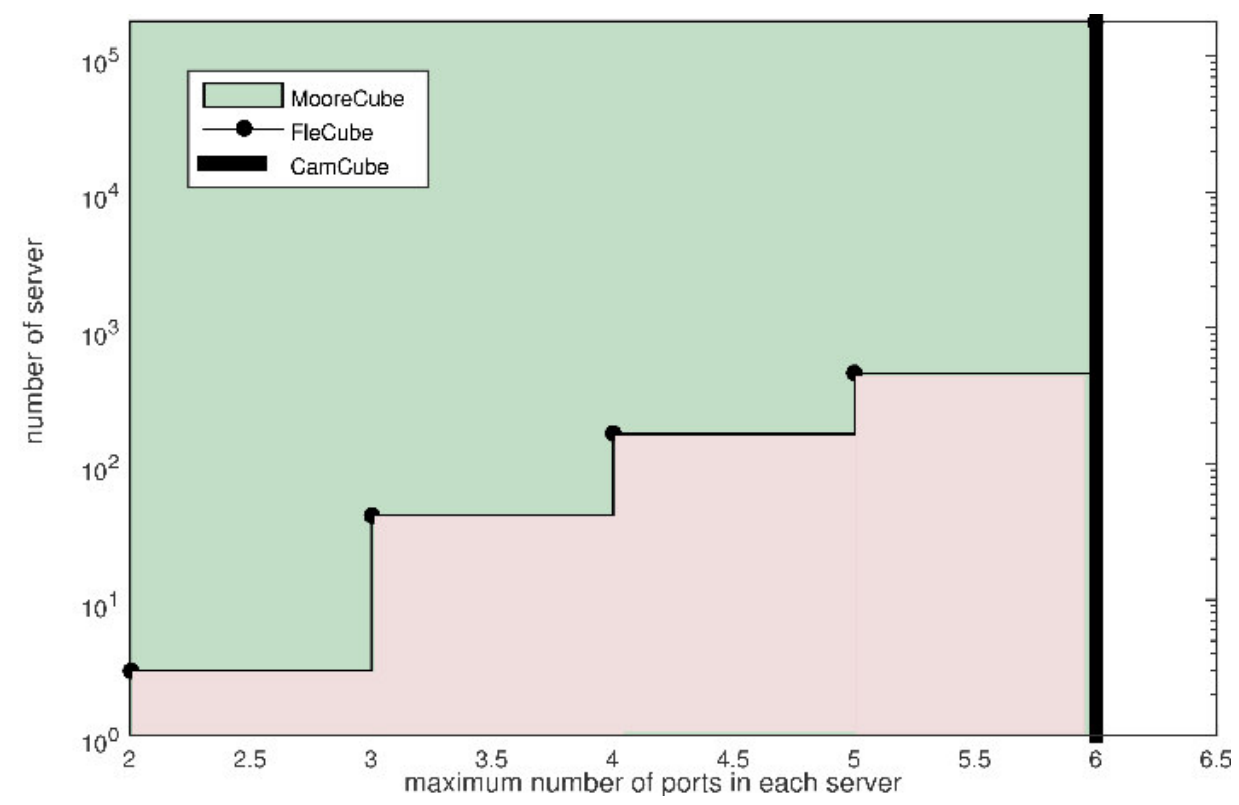

FIG. 6.2. The maximum number of servers used in the number of ports

this port number. So CamCube does not have any flexibility in using server ports. FleCube has more flexibility than CamCube and can use any number of ports. The only problem with this approach is that by choosing the number of required ports, we cannot add new servers more than the certain number that is clear at first and we can calculate at first. For example, with 3-port servers in FleCube, the maximum number of servers that can connect is 42 . So it has less flexibility in using server ports. But MooreCube is more flexible than others and can use any number of server ports.

6.3. Scalability. Scalability means the maximum number of servers used with any given network diameter. In the experiment, network diameter increased from 3 to 33 and in each stage, a maximum number of servers that can connect is calculated. Results are shown in Fig. 6.3.

As indicated in Fig. 6.3, CamCube do not have any scalability with increasing network diameter. FleCube is more scalable than CamCube. But MooreCube is more scalable than CamCube and FleCube. Because uses Moore graph and can construct with any number of server ports.

6.4. Delay in routing. This experiment calculates link delay in sending and receiving the message. Delay is calculated in two modes; with congestion and without congestion. The results are shown in Fig. 6.4.

As indicated in Fig. 6.4, if links have no congestion, the delay is constant and delivery time of packets not change. It means routing algorithm correctly runs and is able to use different routes which are in the routing table. If links have congestion, the delay will be enhanced with increasing flow. The increase in delay is due to static routing in data centers. Static routing, run before using DCN and all of the paths stored in routing tables.

7. Conclusion and future work. In this paper, we proposed MooreCube as a DCN architecture that is scalable and flexible. MooreCube does not use any switch and connects servers directly. So it stores the cost of the purchase and maintenance of the switch and has better performance than switch-centric architectures. MooreCube has more scalability and flexibility and less network diameter. MooreCube uses DPP for routing in DCN. DPP calculate parallel paths depends on server ports. Parallel paths distribute flow between links and prevent network failure.

As future work we intend to use of compound graph as BB to decrease network diameter. This paper focus on scalability and flexibility, as a future work, we want to propose a dynamic parallel path routing that runs simultaneously with the network using. Decreasing delay can be another future work. Producing parallel paths 


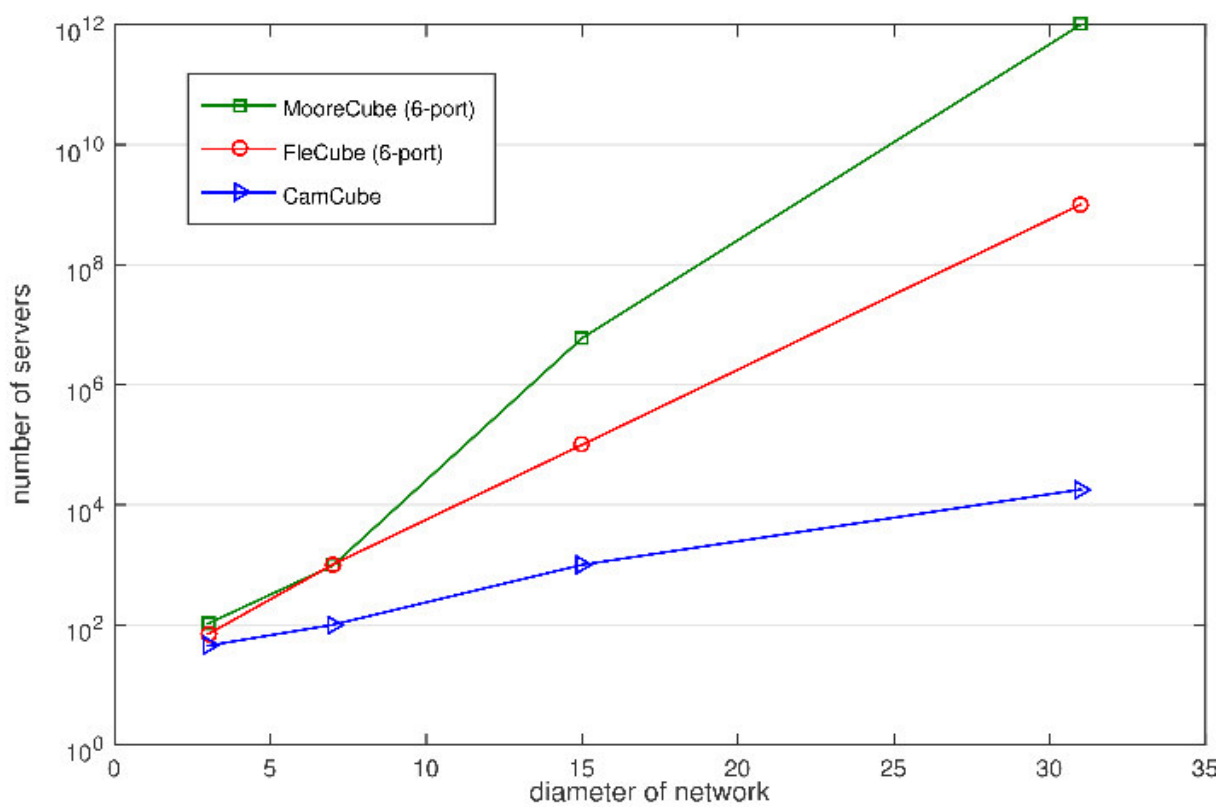

FIG. 6.3. Scalability on network diameter

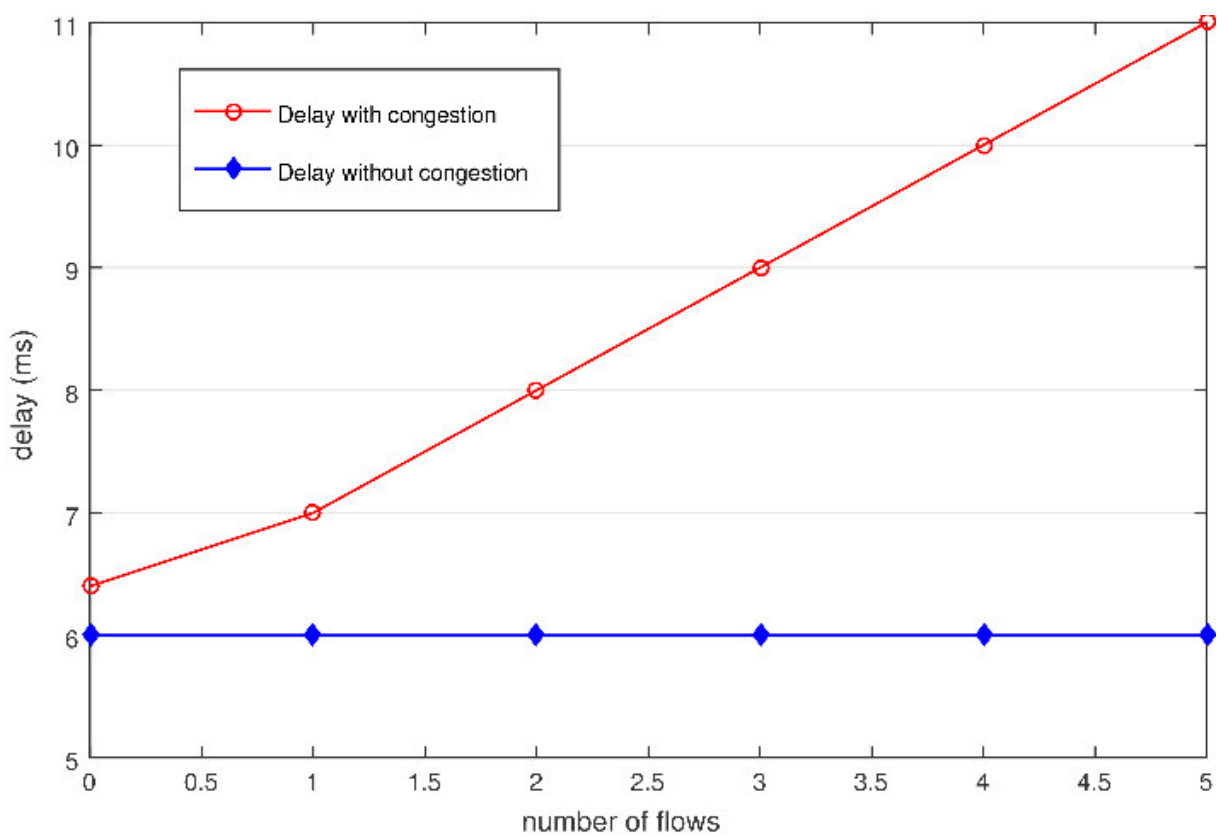

FIG. 6.4. Average delay and number of passed flow in MooreCube

with the same number of links is another future work.

\section{REFERENCES}

[1] W. Tian and Y. ZhaO, Resource modeling and definitions for cloud data centers, Chapter 3, Optimized Cloud Resource Management and Scheduling, 2015, pp. 51-77.

[2] D. Li, Y. Shen And K. Li, FleCube: A flexibly-connected architecture of data center networks on multi-port servers, Computer 
Communications, 70 (2015), pp. 1-10.

[3] B. Wang, z. Qi, R. Ma, H. Guan and V. Vasilakos, A survey on data center networking for cloud computing, Computer Networks, 91 (2015), pp. 528-547.

[4] G. Wu, H. Gu, K. WANG, X. Yu And Y. Guo, A scalable AWG-based data center network for cloud computing, Optical Switching and Networking, 16 (2015), pp. 46-51.

[5] Y. Shen, K. Li, And W. Shi, Advanced topics on cloud computing, Journal of Computer and System Sciences, 79 (2013), pp. 1199, 2013.

[6] T. Wang, Z. Su, Y. XIA, J. Muppala and M. Hamdi, Designing efficient high performance server-centric data center network architecture, Computer Networks, 79 (2015), pp. 283-296.

[7] X. YU, H. GU, Y. YANG AND K. WANG, RingCube An incrementally scale-out optical interconnect for cloud computing data center, Future Generation Computer Systems, 54 (2016), pp. 41-51.

[8] S. Fu, B. Wu, X. Jiang, A. Pattavina, H. Wen and H. Yu, Switch cost and packet delay tradeoff in data center networks with switch reconfiguration overhead, Computer Networks, 87 (2015), pp. 33-43.

[9] M. Uddin, Y. darabidarabkhani, A. Shah and J. Memon, Evaluating power efficient algorithms for efficiency and carbon emissions in cloud data centers: A review, Renewable and Sustainable Energy Reviews, 51 (2015), pp. 1553-1563.

[10] S. Filiposka and C. Juiz, Community-Based complex Cloud data Center, Physica A: Statistical Mechanics and its Applications, 419 (2015), pp. 356-372.

[11] A. Erickson, A. Stewart, J. Navaridas and A. Kiasari, Star-replaced networks: A generalised class of dual-port servercentric data center networks, Computer Networks, 2015, pp. 1-30.

[12] M. Miller And J. Siran, Moore graphs and beyond A survey of the degreediameter problem, University of Newcastle, The Electronic Journal of Combinatorics, 20 (2013), pp. 1-92.

[13] E. DuCEY, On the critical group of the missing Moore graph, Discrete Mathematics, 340 (2017), pp. 1104-1109.

[14] T. Baker, B. Dawsari, H. Tawfik, D. Reid and Y. NGOKo, GreeDi: An energy efficient routing algorithm for big data on cloud, Ad Hoc Networks, 35 (2015), pp. 83-96.

[15] Z. Zhang, W. Hu, T. Ye, W. Sun, L. ZhaO and K. Zhang, Routing and spectrum allocation in multi-ring based data center networks, Optics Communications, 360 (2016), pp. 25-34.

[16] M.A. Fares, A. Loukissas and A. Vahdat, A Scalable, commodity data center network architecture, Acm Sigcomm, 2008, pp. $1-12$.

[17] R. Mysore, A. Pamboris, N. Farrington, N. Huang, P. Miri, S. Radhakrishnan, V. Subramanya and A. Vahdat, PortLand: A Scalable Fault-Tolerant, Sigcomm Proceedings of the ACM Sigcomm, Conference on Data Communication, Newyork, USA, 2009, pp. 39-50.

[18] A. Greenberg, J. Hamilton and N. Jain, VL2: a scalable and flexible data center network, Sigcomm Proceedings of The ACM Sigcomm, Conference on Data Communication, New York, USA, 2009, pp. 51-62.

[19] A. Singla, Ch. Hong, L. Popa And B. Godfrey, Jellyfish: networking data centers randomly, NSDI'12 Proceedings of the 9th USENIX Conference on Networked Systems Design and Implementation, Berkeley, USA, 2012, pp. 1-6.

[20] Y. Yu AND C. QIAn, Space Shuffle: a scalable, flexible and high bandwidth data center network, ICNP '14 Proceeding of the 2014 IEEE 22nd International Conference on Network Protocols, 2014, pp. 13-24.

[21] L. Gyarmati And T. Trinh, Scafida: a scale-free network inspired data center, ACM Sigcomm Computer Communication Review, 40 (2010), pp. 4-12.

[22] M. Csernai, A. Gulyas, A. Korosi, B. Sonkoly and G. Biczok, Incrementally upgradable data center architecture using hyperbolic tessellations, Computer Networks, 57 (2013), pp. 1373-1393.

[23] J. Shin, B. Wong And E. Sirer, Small-World Datacenters, SOCC '11 Proceedings of the 2nd ACM Symposium on Cloud Computing, Newyork, USA, 2011, pp. 1-13.

[24] C. Guo, H. Wu, K. Tan, L. Shi, Y. Zhang and S. Lu, DCell: A Scalable and Fault-Tolerant Network, ACM Sigcomm Computer Communication Review, 38 (2008), pp. 75-86.

[25] C. Guo, G. Lu, D. Li, L. Wu, X. Zhang and Y. Shi, BCube: A high performance, Server-centric Network, ACM Sigcomm Computer Communication Review, 39 (2009), pp. 63-74.

[26] D. Li, H. Wu, K. TAN, Y. Zhang and S. Lu, FiConn: using backup port for server interconnection in data centers, IEEE Infocom, 2009, pp. 2276- 2285.

[27] D. Lin, Y. Liu, M. Hamdi and J. Muppala, FlatNet: towards a flatter data center network, Globecom Next Generation Networking and Internet Symposium, 2012, pp. 2499-2504.

[28] D. Guo, T. Chen, D. Li, M. Li, Y. Lio and G. Chen, Expandable and cost-effective network structures for data centers using dual-port servers, IEEE Transaction on Computers, 62 (2013), pp. 1303-1317.

[29] Y. LiaO, J. Yin, D. Yin AND L. GaO, DPillar: dual-port server interconnection network for large scale data centers, Computer Networks, 56 (2012), pp. 2132-2147.

[30] D. Li AND J. WU, On the design and analysis of Data Center Network architectures for interconnecting dual-port servers, Infocom IEEE Conference on Computer Communication, 2014, pp. 1851-1859.

[31] Li, Deshun, Yanming Shen, And KeqIU Li, FleCube: A flexibly-connected architecture of data center networks on multi-port servers, Computer Communications 77 (2016): 62-71.

[32] A. Gawanmeh,, And A. Alomari, Challenges in formal methods for testing and verification of cloud computing systems, Scalable Computing: Practice and Experience, 16 (2015): 321-332.

[33] D. Guo, C. LI, J. Wu and X. Zhou, DCube: A family of network structures for containerized data centers using dual-port servers, Computer Communications, 53 (2014), pp. 13-25.

[34] D. Li, J. Wu, Z. Liu and F. Zhang, Dual-centric data center network architectures, in Proceedings of IEEE ICPP_15, 2015.

[35] T. Wang, Z. Su, Y. Xia, J. Muppala And M. Hamdi, SprintNet: A high performance server-centric network architecture 
for data centers, Computer Networks, 79 (2015), pp. 283-296.

[36] H. Libdeh, P. Costa, A. Rowstorn, G. Shea And A. Donnelly, cam cube: symbiotic routing in future data centers, ACM Sigcomm Computer Communication Review, 40 (2010), pp. 51-62.

[37] T. Wang, Z. Su, Y. XIA, B. QIn And M. Hamd, NovaCube: A low latency torus-based network architecture for data centers, IEEE Global Communications Conference (Globecom), Austin, TX, 2014, pp. 2252-2257.

[38] A. Hoffman and R. Singleton, On Moore graphs of diameter 2 and 3, IBM Journal of Research and Development, 4 (1960), pp. 497-504.

Edited by: Amjad Gawanmeh

Received: Jul 1, 2017

Accepted: Oct 27, 2017 